\section{Item Analysis}

Michael Franzen

Allegheny General Hospital, Pittsburgh, PA, USA

\section{Cross-References}

- Classical Test Theory

Item Difficulty

- Reliability

\title{
Definition
}

Item analysis is, as the name suggests, the evaluation of an individual item in the context of a test. Item analysis includes examination of item difficulty, reliability, and discrimination, as well as relation of the item to the scale to which it has been assigned. Typically, in classical test theory, the focus has been on the scale score, whereas in item response theory, the emphasis is on the item itself. However, item analysis is an important part of test construction in classical test theory because it guides decisions regarding whether to discard,

\section{References and Readings}

Facon, B., \& Nuchadee, M.-L. (2010). An item analysis of Raven's colored progressive matrices among participants with Down syndrome. Research in Developmental Disabilities, 31, 243-249.

Finch, W. H. (2016). Detection of differential item functioning for more than two groups: A Monte Carlo comparison of methods. Applied Measurement in Education, 29(1), 30-45.

Green, B. F. (1981). A primer of testing. In J. M. Notterman (Ed.), The evolution of psychology: Fifty years of the American Psychologist (pp. 193-212). Washington, DC: American Psychological Association. 